\title{
Arithmetic Triangle
}

\author{
Luís Dias Ferreira ${ }^{1}$ \\ ${ }^{1}$ Colégio Valsassina, Av. Teixeira da Mota, Quinta das Teresinhas, 1959-010 Lisboa, Portugal \\ Correspondence: Luís Dias Ferreira, Av. Teixeira da Mota, Quinta das Teresinhas, 1959-010 Lisboa, Portugal. Tel: 00- \\ 351-210-195-836. E-mail: luisdiasferreira@clix.pt
}

\author{
Received: February 10, 2017 Accepted: March 17, 2017 Online Published: March 21, 2017 \\ doi:10.5539/jmr.v9n2p100～URL: https://doi.org/10.5539/jmr.v9n2p100
}

\begin{abstract}
The product of the first $n$ terms of an arithmetic progression may be developed in a polynomial of $n$ terms. Each one of them presents a coefficient $C_{n k}$ that is independent from the initial term and the common difference of the progression.

The most interesting point is that one may construct an "Arithmetic Triangle", displaying these coefficients, in a similar way one does with Pascal's Triangle. Moreover, some remarkable properties, mainly concerning factorials, characterize the Triangle. Other related 'triangles' - eventually treated as matrices - also display curious facts, in their linear modus operandi, such as successive "descendances".
\end{abstract}

Keywords: arithmetic progression, factorials of order k, arithmetic triangle, descendant matrices, progressive matrix, matrix column product, harmonic numbers.

\section{Introduction}

What is the product of the first $n$ terms of an arithmetic progression? Contrary to geometrical progressions, where simple equations allow to calculate the sum or the product of its first $n$ terms, the issue concerning the product for an arithmetic progression is not so easy. However, its study brings some fascinating results and, to my knowledge, new concepts in Linear Algebra. I propose a little journey on this subject.

First of all, I discovered that it relates to the starting point of a paper of mine, on "Integer Binomial Plan", published in this same journal, in August 2010 (Ferreira), presenting a generalization on factorials and binomial coefficients to all integers. Since then I never returned to the subject till recently, when a student asked me if I knew a formula for the product of the first $n$ terms of an arithmetic progression:

$$
a_{1}, a_{2}=a_{1}+d, a_{3}=a_{1}+2 d, \ldots, a_{n}=a_{1}+(n-1) d, \ldots .
$$

I didn't; but then we began thinking on the problem. Let $P_{n}=a_{1} \cdot a_{2} \cdot \ldots \cdot a_{n}$. Following a simple procedure, remarking that $P_{n}=P_{n-1} \cdot\left[a_{1}+(n-1) d\right]$ and beginning with $P_{1}=a_{1}$, we soon found out that $P_{n}$ may be expressed by a sum of $n$ terms concerning powers of $a_{1}$ and $d$ multiplied by $n$ coefficients, which are independent from those variables. We followed the procedure up to $n=5$ :

$$
\begin{aligned}
& P_{1}=1 \cdot a_{1} \\
& P_{2}=1 \cdot a_{1}^{2}+1 \cdot a_{1} d \\
& P_{3}=1 \cdot a_{1}^{3}+3 \cdot a_{1}^{2} d+2 \cdot a_{1} d^{2} \\
& P_{4}=1 \cdot a_{1}^{4}+6 \cdot a_{1}^{3} d+11 \cdot a_{1}^{2} d^{2}+6 \cdot a_{1} d^{3} \\
& P_{5}=1 \cdot a_{1}^{5}+10 \cdot a_{1}^{4} d+35 \cdot a_{1}^{3} d^{2}+50 \cdot a_{1}^{2} d^{3}+24 \cdot a_{1} d^{4},
\end{aligned}
$$

so we could establish the coefficients triangle 
This reminds Newton's Binomial and Pascal's Triangle. The question which naturally arises is: may one built this "Arithmetic Triangle" in a similar way one does with binomial coefficients? To eventually achieve this goal, one must find and understand the pattern underneath, which is not self-evident. But the answer to the question is affirmative. In fact, later that day, I found out that we may indeed construct the triangle progressively, in a similar way we do with Pascal's triangle. To my surprise, however, this issue brought back complete and incomplete factorials.

The search for an answer and some collateral results are the subject of this article.

I must point out that - also surprisingly - the solution found corresponds closely to the unsigned Stirling numbers of the first kind, which I didn't know. Several papers have been recently produced concerning Stirling numbers and cyclic groups, for instance (Broder, 1984) and (Deveci \& Akuzum, 2014); or Pascal matrices (Deveci \& Karaduman, 2012) and (Hiller, 2016). But they don't seem especially relevant to this article, which essentially deals with basic stuff.

\subsection{Factorial of Order $\mathrm{k}$}

Consider a real number $x$ and an integer $k$, along with the special case $0 !=1$; the $\boldsymbol{x}$ factorial of order $\boldsymbol{k}$ is defined by (read " $x$ k factorial")

$$
x_{(k)} !=\frac{x !}{(x-k) !},
$$

or, for $k>0$,

$$
x_{(k)} !=\prod_{i=1}^{k}(x-i+1)=x \cdot(x-1) \cdot \ldots \cdot(x-k+1) ;
$$

in this case, $x_{(k)}$ ! is an 'incomplete factorial', the product of the $k$ major values of $x$ !.

We assume that the definition above may be extended to negative order numbers; but then we conclude that

$$
x_{(-k)} !=\frac{1}{\left.(x+k)_{(k)}\right)} .
$$

Remark that

$$
\begin{cases}x_{(0)} !=1 & \\ x_{(x)} !=x ! & \\ x_{(1)} !=x, & \text { for } x \neq 0 \\ x_{(-1)} !=\frac{1}{x+1}, & \text { for } x \neq-1 .\end{cases}
$$

This proofs to be quite consistent. Generally speaking, as I explained in (Ferreira), factorials of order $k$ must be seen as limits of a quotient. Besides, as presupposed in the generalization of $x_{(k)}$ ! from positive to negative numbers $x$, if $k>0$ this factorial represents the product of the $k$ values presented in equation (2.a) even for $x<0$, where $x !=\infty$. For instance,

$$
(-8)_{(3)} !=\frac{(-8) !}{(-11) !}=\frac{(-8) \cdot(-9) \cdot(-10) \cdot(-11) !}{(-11) !}=(-8) \cdot(-9) \cdot(-10)=-720 ;
$$

if $k<0$, then, according to (3), $n_{(k)}$ ! represents the inverse of a product of $k$ factors:

$$
(-8)_{(-3)} !=\frac{1}{(-5)_{(3)} !}=\frac{1}{(-5) \cdot(-6) \cdot(-7)}=-\frac{1}{210} .
$$

In these terms, of course

$$
\left(\begin{array}{c}
n \\
k
\end{array}\right)=\frac{n_{(k)} !}{k !}
$$

is a generalized binomial coefficient and so, the development in MacLaurin series of the function $y=(1+x)^{n}$ may be written as

$$
(1+x)^{n}=\sum_{k=0}^{+\infty}\left(\begin{array}{l}
n \\
k
\end{array}\right) \cdot x^{k}=\sum_{k=0}^{+\infty} \frac{n_{(k)} !}{k !} x^{k} .
$$

\subsection{Rising and Falling Factorials}

In a rapid web research, these days, I found out that the concept of $x_{(k)}$ ! was already known, with other name and symbol(s), since the end of the 19th Century; it has been introduced by Leo A. Pochhammer with the notation $(x)_{k}$. I was quite surprised but, anyway, Pochhammer doesn't consider negative integers for $k$. 
I also discovered the useful concept of rising factorial - in contrast with falling factorial, this is, $x_{(k)} !-$, which I'll note by $x^{(k)}$ ! ; for $x \neq 0$ and positive integer $k$ :

$$
x^{(k)} !=\prod_{i=1}^{k}(x+i-1)=x \cdot(x+1) \cdot \ldots \cdot(x+k-1) .
$$

It's easy to see that

$$
x^{(k)} !=(x+k-1)_{(k)} ! \quad \text { or } \quad x_{(k)} !=(x-k+1)^{(k)} ! ;
$$

For instance, $7^{(4)} !=10_{(4)} !=7 \cdot 8 \cdot 9 \cdot 10$. Based on the assumption that the equality (5.a) must stand for any values of $x$ and $k$ and keeping in mind the equation (2), we'll generically define the rising factorial $x^{(k)}$ ! by

$$
x^{(k)} !=\frac{(x+k-1) !}{(x-1) !}
$$

Therefore, as particular cases, we obtain

$$
\left\{\begin{array}{l}
x^{(0)} !=1 \\
1^{(k)} !=k !
\end{array}\right.
$$

Besides, we'll consider (5.a) also valid for negative order number $-k$; but then, according to (3):

$$
x^{(-k)} !=\frac{1}{(x-1)_{(k)} !} .
$$

Finally: to simplify, we may read $x_{(k)}$ ! and $x^{(k)}$ ! respectively as " $\boldsymbol{x}$ down $\boldsymbol{k}$ factorial" and " $\boldsymbol{x}$ up $\boldsymbol{k}$ factorial".

\subsection{New Generalization on Factorials}

Let's resume the arithmetic progression

$$
a_{1}=a, a_{2}=a_{1}+d, a_{3}=a_{1}+2 d, \ldots, a_{n}=a_{1}+(n-1) d, \ldots,
$$

where $a_{1}$ is the initial term. The constant $d$ is usually known as the common difference of successive members; I will call it the increment of the progression.

Let $P_{n}$ be the product of the first $n$ terms of this arithmetic progression. It's quite evident that:

- for $a_{1}=d=1: P_{n}=n !$;

We'll say that this is an unitary arithmetic progression (u.a.p.) on basis 1 (its initial term).

- for $a_{1}=a$ and $d=1: P_{n}=a^{(n)} !=(a+n-1)_{(n)} !$.

Now, we may put the $n$-th term of the progression above in the form

$$
a_{n}=d\left[\frac{a}{d}+(n-1)\right]
$$

and the expression between brackets corresponds to an u.a.p. on basis $a / d$. In this case,

$$
P_{n}=\prod_{i=1}^{n} a_{i}=d^{n} \prod_{i=1}^{n}\left[\frac{a}{d}+(i-1)\right]
$$

and we'll identify $P_{n}$ with the $\boldsymbol{a}$ rising factorial of increment $\boldsymbol{d}$ and order $\boldsymbol{n}$, noted by $a^{(d ; n)}$ ! (read " $\boldsymbol{a}$ up $\boldsymbol{d} \boldsymbol{n}$ factorial"):

$$
a^{(d ; n)} !=d^{n}\left(\frac{a}{d}\right)^{(n)} !
$$

This relates to the $\boldsymbol{a}$ falling factorial of increment $\boldsymbol{d}$ and order $\boldsymbol{n}$, noted by $a_{(\boldsymbol{d} ; n)}$ ! (read "a down $\boldsymbol{d} \boldsymbol{n}$ factorial") as follows:

$$
a_{(d ; n)} !=d^{n}\left(\frac{a}{d}\right)_{(n)} !
$$


in such a way that

$$
a_{(d ; n)} !=[a-(n-1) d]^{(d ; n)} ! \quad \text { and } \quad a^{(d ; n)} !=[a+(n-1) d]_{(d ; n)} !,
$$

As a particular case, from (8):

$$
a=d \quad \Rightarrow \quad a^{(a ; n)} !=a^{n} 1^{(n)} !=a^{n} n ! .
$$

Of course, making $d=1$, we recover the simple rising and falling factorials $a^{(1 ; n)} !=a^{(n)} !$ and $a_{(1 ; n)} !=a_{(n)} !$.

\section{Arithmetic Coefficients}

The key point to discover the rule for building the Arithmetic Triangle is to notice that, because of the linear nature of an arithmetic progression, each $P_{n}$ proceeds from the precedent $P_{n-1}$ in a similar linear way.

Our goal is to discover the $n$ coefficients $C_{n k}$ (or $C_{n ; k}$ ) that allow us to write, making $a_{1}=a$, the product $P_{n}$ as

$$
P_{n}=\sum_{k=1}^{n} C_{n k} a^{n-k+1} d^{k-1}=C_{n 1} a^{n} d^{0}+C_{n 2} a^{n-1} d^{1}+\cdots+C_{n k} a^{n-k+1} d^{k-1}+\cdots+C_{n n} a^{1} d^{n-1} .
$$

We'll call these $C_{n k}$ the arithmetic coefficients for the product $P_{n}$. Since the coefficients $C_{n k}$ are independent from $a$ and $d$, we'll make $d=1$ in the following deduction [the numbers in bold correspond to $n-1$ in each case].

$$
\begin{aligned}
P_{1}=a & \Rightarrow \begin{array}{l}
C_{11}=1 . \\
P_{2}=C_{11} a \cdot(a+\underbrace{n-1}_{1})=C_{11} a^{2}+\mathbf{1} C_{11} a
\end{array} \\
P_{3}=\left(C_{21} a^{2}+C_{22} a\right) \cdot(a+\underbrace{n-1}_{2}) & \Rightarrow\left\{\begin{array}{l}
C_{21}=C_{11}=1 \\
C_{22}=(n-1) C_{11}
\end{array}\right. \\
=C_{21} a^{3}+\left(\mathbf{2} C_{21}+C_{22}\right) a^{2}+\mathbf{2} C_{22} a & \Rightarrow\left\{\begin{array}{l}
C_{31}=C_{21}=1 \\
C_{32}=(n-1) C_{21}+C_{22} \\
C_{33}=(n-1) C_{22}
\end{array}\right. \\
P_{4}=\left(C_{31} a^{3}+C_{32} a^{2}+C_{33} a\right) \cdot(a+\underbrace{n-1}_{3}) & \Rightarrow\left\{\begin{array}{l}
C_{42}=(n-1) C_{31}+C_{32} \\
C_{43}=(n-1) C_{32}+C_{33} \\
C_{44}=(n-1) C_{33} .
\end{array}\right.
\end{aligned}
$$

This is enough to recognize the pattern. We see that $C_{n 1}=1$, for every $n$, and we obtain the general rule:

$$
\left\{\begin{array}{l}
C_{n 1}=1 \\
C_{n k}=(n-1) C_{n-1 ; k-1}+C_{n-1 ; k} \\
C_{n n}=(n-1) C_{n-1 ; n-1}, \text { for } n>1 .
\end{array}\right.
$$

A more formal demonstration follows the same path, as we'll see in the sequence. As a matter of fact, the single line

$$
C_{n k}=(n-1) C_{n-1 ; k-1}+C_{n-1 ; k}
$$

is sufficient if we understand that it must be

$$
C_{n k}=0 \text { for } k<1 \text { or } k>n .
$$

This is because $k<1$ corresponds to the 'left wing' - this is, the 'negative-increment' terms - and $k>n$ to upper terms in the progression; in both cases, these terms are out of the product $P_{n}$. The last condition also corresponds to the empty spaces in the Arithmetic Triangle.

Furthermore, we'll generalize $C_{n 1}=1$ to $n=0$, resulting $C_{11}=\underbrace{0 \cdot C_{00}}_{0}+\underbrace{C_{01}}_{1}=1$. 
Theorem 1. If $P_{n}$ is the product of the first $n$ terms of the arithmetic progression given by $a_{k}=a_{1}+(n-1) k$, then

$$
P_{n}=\sum_{k=1}^{n} C_{n k} a^{n-k+1} d^{k-1} \quad \text { with } \quad C_{n k}=(n-1) C_{n-1 ; k-1}+C_{n-1 ; k}
$$

Proof. It's a proof by induction:

1) The equation above is obviously valid for $P_{1}=1 \cdot a$.

2) Let $P_{n-1}=\sum_{k=1}^{n-1} C_{n-1 ; k} a^{n-k} d^{k-1}$.

3) Then, $P_{n}=P_{n-1} \cdot[a-(n-1) d]$

For each $k$ in the resulting sum, we'll have two terms:

$$
C_{n-1 ; k} a^{n-k+1} d^{k-1}+C_{n-1 ; k} a^{n-k} d^{k}
$$

So, if we make $k=i-1$ and $k=i$, we'll get

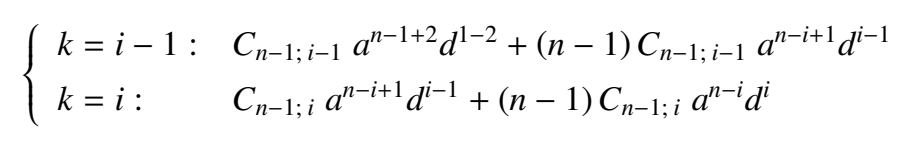

Remark that the second term for $(i-1)$ combines with the first one for $i$ :

$$
\left[(n-1) C_{n-1 ; i-1}+C_{n-1 ; i}\right] a^{n-i+1} d^{i-1}
$$

This is valid for every $i$, therefore for every $k$; but the indexes of $C_{n k}$ relates to the product $a^{n-k+1} d^{k-1}$ and, so, the expression between brackets corresponds to $C_{n k}$, which means that $C_{n k}=(n-1) C_{n-1 ; k-1}+C_{n-1 ; k}$.

Based on (13) or (11), one can progressively construct the Arithmetic Triangle:

$$
\begin{array}{lcccccccccc}
\mathrm{n}=1 & 1 & & & & & & & & & \\
\mathrm{n}=2 & 1 & 1 & & & & & & & & \\
\mathrm{n}=3 & 1 & 3 & 2 & & & & & & & \\
\mathrm{n}=4 & 1 & 6 & 11 & 6 & & & & & & \\
\mathrm{n}=5 & 1 & 10 & 35 & 50 & 24 & & & & & \\
\mathrm{n}=6 & 1 & 15 & 85 & 225 & 274 & 120 & & & & \\
\mathrm{n}=7 & 1 & 21 & 175 & 735 & 1624 & 1764 & 720 & & & \\
\mathrm{n}=8 & 1 & 28 & 322 & 1960 & 6769 & 13132 & 13068 & 5040 & & \\
\mathrm{n}=9 & 1 & 36 & 546 & 4536 & 22449 & 67284 & 118124 & 109584 & 40320 & \\
\mathrm{n}=10 & 1 & 45 & 870 & 9450 & 63273 & 269325 & 723680 & 1172700 & 1026576 & 362880 \\
\ldots & \ldots & \ldots & \ldots & \ldots & \ldots & \ldots & \ldots & \ldots & \ldots & \ldots
\end{array}
$$

Each set of Arithmetic Coefficients - this is, for each value of $n$ - displays the following remarkable properties:

- $\sum_{k=1}^{n} C_{n k}=n !$

- $C_{n n}=(n-1) !$

- For $n>1: \sum_{k=1}^{n-1} C_{n k}=n !-(n-1) !=(n-1) \cdot(n-1) !$

- For $n>1: \sum_{k=1}^{n}(-1)^{(k-1)} C_{n k}=0$.

The first equality is self-evident because, as we have seen, making $a_{1}=d=1$ (u.a.p. on basis 1 ), $P_{n}$ is the product of the $n$ integers from 1 to $n$, this is, $n$ factorial. The second comes from a recurrence relation: from (13) and (12), remembering 
that $C_{11}=1=0$ !, we get, for $n>1$, since $C_{n-1 ; n}=0: \quad C_{n n}=(n-1) C_{n-1 ; n-1}$; so,

$$
\begin{aligned}
& C_{22}=1 \cdot C_{11}=1 ! \\
& C_{33}=2 \cdot C_{22}=2 \cdot 1 !=2 ! \\
& C_{44}=3 \cdot C_{33}=3 \cdot 2 !=3 ! \\
& \ldots \\
& C_{n n}=(n-1) \cdot C_{n-1 ; n-1}=(n-1) ! .
\end{aligned}
$$

The third equality results from combining the previous two. Finally, the fourth equality - which we may call an alternate sum - comes from the fact that, if we consider the 'left wing' of the u.a.p. on basis 1 (by simply making $d=-1$ ), it results $a_{2}=0$ and, so, for $n>1$, each product $P_{n}$ includes the zero.

For instance, making $n=6$ :

- $\sum_{k=1}^{6} C_{6 k}=1+15+85+225+274+120=720=6$ !

- $C_{66}=5 !=120$

- $\sum_{k=1}^{5} C_{6 k}=1+15+85+225+274=600=5 \cdot 5$ !

- $\sum_{k=1}^{6}(-1)^{(k-1)} C_{6 k}=1-15+85-225+274-120=0$.

An important feature here is that the use of the Arithmetic Coefficients transform products, $n$ !, into sums, both with $n$ terms. Concerning these coefficients, here's another interesting fact:

- $\prod_{k=1}^{n} C_{k k}=\prod_{k=1}^{n-1}(n-k) !=\prod_{k=1}^{n-1} k !$.

This is the product of the diagonal terms of the Arithmetic Triangle up from 1 to $n$. Following Neil Sloane and Simon Plouffe, it may be useful to define, for a positive integer $n$, the $n$ superfactorial - I'll note $n !^{*}$ instead of $\operatorname{sf}(n)-$ as

$$
n !^{*}=n !(n-1) !^{*}=\prod_{k=1}^{n} k !=n !(n-1) !(n-2) ! \ldots 2 ! 1 !,
$$

which is equivalent to

$$
n !^{*}=\prod_{k=1}^{n} k^{n-k+1}=n \cdot(n-1)^{2} \cdot(n-2)^{3} \ldots 2^{n-1} \cdot 1^{n} .
$$

This last equality also results from a recurrence relation; informally:

$$
\begin{aligned}
& 2 !^{*}=2 ! 1 ! \\
& 3 !^{*}=\underbrace{3 !}_{3 \cdot 2 !} 2 ! 1 !=3 \cdot(2 !)^{2} 1 !=3 \cdot(\underbrace{2 !}_{2 \cdot 1 !})^{2} 1 !=3 \cdot 2^{2} \cdot 1^{3} \\
& 4 !^{*}=\underbrace{4 !}_{4 \cdot 3 !} 3 !^{*}=4(3 \cdot 2 \cdot 1)\left(3 \cdot 2^{2} \cdot 1^{3}\right)=4 \cdot 3^{2} \cdot 2^{3} \cdot 1^{4} \\
& \ldots \\
& n !^{*}=\underbrace{n !}_{n \cdot(n-1) !}(n-1) !^{*}=n(n-1) !(n-1) !^{*}=n \cdot(n-1)^{2} \cdot(n-2)^{3} \ldots 2^{n-1} \cdot 1^{n} .
\end{aligned}
$$

We may write, then: $\prod_{k=1}^{n} C_{k k}=(n-1) !^{*}$

For instance, for $n=6: \prod_{k=1}^{6} C_{k k}=5 !^{*}=5 \cdot 4^{2} \cdot 3^{3} \cdot 2^{4} \cdot 1^{5}=34560$.

Consider now some other cases for the arithmetic progression:

1. $a=d: a^{n-k+1} d^{k-1}=a^{n}$ and $P_{n}=a^{n} \sum_{k=1}^{n} C_{n k}=a^{n} n !$.

2. As a particular case, $a=d=-1: P_{n}=(-1)^{n} \sum_{k=1}^{n} C_{n k}=(-1)^{n} n !=(-1)_{(n)} !=(-n)^{(n)}$ ! according to Theorem 2 in (Ferreira) and equation (5.a) above. 
3. As another particular case, $a=d=2$ :

$$
\begin{array}{lllllllll}
2 & 4 & 6 & 8 & 10 & \ldots & 2 k & \ldots
\end{array}
$$

this is the set of even numbers and it results $2^{(2 ; n)} !=(2 n) ! !=P_{n}=2^{n} \sum_{k=1}^{n} C_{n k}=2^{n} n !$ [where $(2 n) ! !$ is the double factorial of $m=2 n]$.

For instance, $8 ! !=P_{4}=2^{4}(1+6+11+6)=2^{4} 4 !=16 \cdot 24=384$.

4. $a=1$ and $d=2$ :

$\begin{array}{lllllllll}1 & 3 & 5 & 7 & 9 & \ldots & 2 k-1 & \ldots\end{array}$

this is now the set of odd numbers and it results $1^{(2 ; n)} !=(2 n-1) ! !=P_{n}=\sum_{k=1}^{n} 2^{k-1} C_{n k}$ [where $(2 n-1) ! !$ is the double factorial of $m=2 n-1]$.

For instance, $9 ! !=P_{5}=1 \cdot 1+2 \cdot 10+4 \cdot 35+8 \cdot 50+16 \cdot 24=1+20+140+400+384=945$.

5. Making $d=1: P_{n}=\sum_{i=1}^{n} C_{n i} a^{n-i+1}=a_{n(n)} !=a^{(n)} !$, for a given $a$ or $a_{n}=a+n-1$.

For instance, making $a_{5}=10$, then $a=6$, this corresponding to the progression

$$
\begin{aligned}
& \begin{array}{llllllll}
6 & 7 & 8 & 9 & 10 & 11 & \ldots
\end{array} \\
& \text { then } P_{5}=\sum_{i=1}^{5} C_{5 i} \cdot 6^{6-i}=7776+12960+7560+1800+144=30240=10_{(5)} !=6^{(5)} \text { ! }
\end{aligned}
$$

\section{Direct Determination of Arithmetic Coefficients}

\subsection{Sum-factorials}

My goal here was to establish an explicit equation (or equations) allowing to directly obtain whatever coefficient $C_{n k}$, independently from the Arithmetic Triangle (as one does with binomial numbers). Unfortunately, I haven't been able to induce a simple formula; in the end, for $k>4$, we stumble on a recursive method of the kind. Anyway, some interesting results appear. So, first of all, it is convenient to define the $\boldsymbol{x}$ sum-factorial (also known as " $x$-th triangular number") as

$$
x \dagger=\sum_{i=1}^{x} i=1+2+3+\cdots+(x-1)+x=\frac{(x+1)_{(2)} !}{2},
$$

or $x \dagger=x+(x-1) \dagger$, together with the special case $0 \dagger=0$; and, for a non-negative integer $k$, the $\boldsymbol{x}$ sum-factorial of order $\boldsymbol{k}$ as

$$
\begin{aligned}
x_{(k)} \dagger & =x \dagger-(x-k) \dagger \\
& =\sum_{i=1}^{k}(x-i+1)=x+(x-1)+\cdots+(x-k+1) \\
& =\frac{k}{2}(2 x-k+1),
\end{aligned}
$$

this is, the sum of the $k$ major values of $x \dagger$. As for product-factorials $x_{(k)}$ !, we'll consider that the definition of $x_{(k)} \dagger$ also stands for negative integers $-k$; the result is

$$
\begin{aligned}
x_{(-k)} \dagger & =-[(x+k) \dagger-x \dagger] \\
& =-(x+k)_{(k)} \dagger \\
& =-\frac{k}{2}(2 x+k+1) .
\end{aligned}
$$

It's easy to see that, as particular cases,

$$
\left\{\begin{array}{l}
x_{(0)} \dagger=0 \\
x_{(x)} \dagger=x \dagger \\
x_{(1)} \dagger=x \\
x_{(-1)} \dagger=-(x+1)
\end{array}\right.
$$

The infinite sequence of $u_{j}=j \dagger$ is

$$
\begin{array}{lllllllllllll}
1 & 3 & 6 & 10 & 15 & 21 & 28 & 36 & 45 & 55 & 66 & 78 & \ldots
\end{array}
$$

Finally, as a curiosity, if we consider $x_{(k)} \dagger$ as a falling sum-factorial, then the $\boldsymbol{x}$ rising sum-factorial is

$$
\begin{aligned}
x^{(k) \dagger} & =\sum_{i=1}^{k}(x+i-1)=x+(x+1)+\cdots+(x+k-1) \\
& =\frac{k}{2}(2 x+k-1) .
\end{aligned}
$$




\subsection{Obtaining Arithmetic Coefficients}

Coming back to the $C_{n k}$ issue, rather than providing exhaustive proofs, we'll take an inductive path. This, I think, is enough for the moment and more instructive. We'll proceed step by step, each column at a time, increasing the value of $k$, always keeping in mind that $C_{n k}=0$ for $k>n$ and the recursive relation

$$
C_{n k}=(n-1) C_{n-1 ; k-1}+C_{n-1 ; k} .
$$

1. First of all, we know that $C_{n 1}=1$. Then, it's easy to conclude that, for $k=2$ :

$$
\left\{\begin{array}{l}
C_{22}=1+0=1 \\
C_{32}=2 C_{21}+C_{22}=2+1 \\
C_{42}=3 C_{31}+C_{32}=3+2+1 \\
C_{52}=4 C_{41}+C_{42}=4+3+2+1 \\
\ldots
\end{array}\right.
$$

this is,

$$
C_{n 2}=(n-1) \dagger=\frac{n-1}{2}(1+n-1) \quad \Rightarrow \quad C_{n 2}=\frac{1}{2} n_{(2)} !=\left(\begin{array}{l}
n \\
2
\end{array}\right) .
$$

2. We'll proceed in the same way for $k=3$ :

$$
\left\{\begin{array}{l}
C_{23}=0 \\
C_{33}=2 C_{22}+C_{23}=\frac{1}{2} 2 \cdot 2_{(2)} ! \\
C_{43}=3 C_{32}+C_{33}=\frac{1}{2}\left[3 \cdot 3_{(2) !}+2 \cdot 2_{(2)} !\right] \\
C_{53}=4 C_{42}+C_{43}=\frac{1}{2}\left[4 \cdot 4_{(2)} !+3 \cdot 3_{(2)} !+2 \cdot 2_{(2)} !\right] \\
\ldots,
\end{array}\right.
$$

this is,

$$
C_{n 3}=\frac{1}{2} \sum_{j=2}^{n-1} j \cdot j_{(2)} !=\sum_{j=2}^{n-1} F_{j},
$$

where

$$
F_{j}=\frac{1}{2} j \cdot j_{(2)} !=j \cdot\left(\begin{array}{c}
j \\
2
\end{array}\right) .
$$

These are the values for the $F_{j}, j$ from 1 to 12 :

$$
\begin{array}{llllll}
F_{1}=0 & F_{2}=2 & F_{3}=9 & F_{4}=24 & F_{5}=50 & F_{6}=90 \\
F_{7}=142 & F_{8}=224 & F_{9}=324 & F_{10}=450 & F_{11}=605 & F_{12}=792 .
\end{array}
$$

So, for instance, $C_{73}=\sum_{j=2}^{6} F_{j}=2+9+24+50+90=175$.

3. Let's examine now the case of $k=4$ :

$$
\left\{\begin{array}{l}
C_{34}=0 \\
C_{44}=3 C_{33}+C_{34}=3 F_{2} \\
C_{54}=4 C_{43}+C_{44}=4 F_{3}+(4+3) F_{2}=4_{(1)} \dagger \cdot F_{3}+4_{(2)} \dagger \cdot F_{2} \\
C_{64}=5 C_{53}+C_{54}=5_{(1)} \dagger \cdot F_{4}+5_{(2)} \dagger \cdot F_{3}+5_{(3)} \dagger \cdot F_{2} \\
\ldots,
\end{array}\right.
$$

this is,

$$
C_{n 4}=\sum_{i=1}^{n-3}(n-1)_{(i)} \dagger \cdot F_{n-i-1} .
$$

We'll write this as

$$
C_{n 4}=\sum_{i=1}^{n-3} A_{n 4}^{i} \cdot F_{n-i-1}
$$


making

$$
A_{n 4}^{i}=(n-1)_{(i)} \dagger=\frac{i}{2}(2 n-i-1) .
$$

where $i$ is not an exponent but an upper index. Remark that

$$
A_{n 4}^{0}=0, \quad A_{n 4}^{1}=n-1 \quad \text { and, for } n>4, \quad A_{n 4}^{i}=A_{n 4}^{i-1}+(n-i) .
$$

The values for $A_{n 4}^{i}$ (where $\left.1 \leq i \leq n-3\right)$ are:

$\begin{array}{lcccccccccc}\mathrm{i} & 1 & 2 & 3 & 4 & 5 & 6 & 7 & 8 & 9 & \ldots \\ \mathrm{n}=4 & 3 & & & & & & & & & \\ \mathrm{n}=5 & 4 & 7 & & & & & & & & \\ \mathrm{n}=6 & 5 & 9 & 12 & & & & & & & \\ \mathrm{n}=7 & 6 & 11 & 15 & 18 & & & & & & \\ \mathrm{n}=8 & 7 & 13 & 18 & 22 & 25 & & & & & \\ \mathrm{n}=9 & 8 & 15 & 21 & 26 & 30 & 33 & & & & \\ \mathrm{n}=10 & 9 & 17 & 24 & 30 & 35 & 39 & 42 & & & \\ \mathrm{n}=11 & 10 & 19 & 27 & 34 & 40 & 45 & 49 & 52 & & \\ \mathrm{n}=12 & 11 & 21 & 30 & 38 & 45 & 51 & 56 & 60 & 63 & \\ \ldots & \ldots & \ldots & \ldots & \ldots & \ldots & \ldots & \ldots & \ldots & \ldots & \ldots\end{array}$

For instance, making $n=8$, we get

$$
\begin{aligned}
C_{84} & =\sum_{i=1}^{5} A_{84}^{i} \cdot F_{n-i-1} \\
& =A_{84}^{1} \cdot F_{6}+A_{84}^{2} \cdot F_{5}+A_{84}^{3} \cdot F_{4}+A_{84}^{4} \cdot F_{3}+A_{84}^{5} \cdot F_{2} \\
& =7 \cdot 90+13 \cdot 50+18 \cdot 24+22 \cdot 9+25 \cdot 2=1960 .
\end{aligned}
$$

OBS.: we'll necessarily note $C_{n ; k}$ or $A_{n ; k}^{i}$ in the case of $n$ or $k$ being two digits numbers, like $A_{12 ; 4}^{5}=45$.

4. Following a similar path, we'll come now to $k=5$; and then generalize the result to $k \geq 4$.

$$
\left\{\begin{array}{l}
C_{45}=0 \\
C_{55}=4 C_{44}+C_{45}=4 A_{44}^{1} \cdot F_{2} \\
C_{65}=5 C_{54}+C_{55}=5 A_{54}^{1} F_{3}+\left(5 A_{54}^{2}+4 A_{44}^{1}\right) F_{2} \\
C_{75}=6 C_{64}+C_{65}=6 A_{64}^{1} F_{4}+\left(6 A_{64}^{2}+5 A_{54}^{1}\right) F_{3}+\left(6 A_{64}^{3}+5 A_{54}^{2}+4 A_{44}^{1}\right) F_{2} \\
\ldots
\end{array}\right.
$$

Take for instance, $n=7$; if we put

$$
\left\{\begin{array}{l}
A_{75}^{1}=6 A_{64}^{1} \\
A_{75}^{2}=6 A_{64}^{2}+5 A_{54}^{1} \\
A_{75}^{3}=6 A_{64}^{3}+5 A_{54}^{2}+4 A_{44}^{1},
\end{array}\right.
$$

this corresponding to diagonal product/sums of $i$ terms in the table (25), we'll write

$$
C_{75}=A_{75}^{1} \cdot F_{4}+A_{75}^{2} \cdot F_{3}+A_{75}^{3} \cdot F_{2},
$$

which is an analogous equation to (23.a).

We deal here with a recursive process; so, for $k \geq 5$, it must be

$$
A_{n k}^{i}=\sum_{j=1}^{i}(n-j) A_{n-j ; k-1}^{i-j+1} .
$$


Remark that the number of terms for $C_{n k}$ is always equal to $n-k+1$; on the other hand, the smallest term of the $F_{b}$ is $F_{2}$ and, so, the biggest one is $F_{n-k+2}$. We are therefore able to write a general recursive equation, for $k \geq 4$ :

$$
\begin{aligned}
C_{n k} & =\sum_{i=1}^{n-k+1} A_{n k}^{i} \cdot F_{(n-k+3)-i} \\
& =A_{n k}^{1} \cdot F_{n-k+2}+A_{n k}^{2} \cdot F_{n-k+1}+\cdots+A_{n k}^{n-k+1} \cdot F_{2} .
\end{aligned}
$$

Because of its recursive nature, this is mainly of theoretical interest, from an algebraic point of view. Its application to real cases becomes quite fastidious, as $k$ increases. Consider, for instance, the above-mentioned case $C_{75}$; the first step is easy, deriving directly from table (25):

$$
\left\{\begin{array}{l}
A_{75}^{1}=6 \cdot 5=30 \\
A_{75}^{2}=6 \cdot 9+5 \cdot 4=74 \\
A_{75}^{3}=6 \cdot 12+5 \cdot 7+4 \cdot 3=119
\end{array} \Rightarrow C_{75}=30 \cdot 48+74 \cdot 9+119 \cdot 2=1624 .\right.
$$

Now, for $k=6$, to compute the two $A_{76}^{i}$, we are obliged to previously calculate $A_{65}^{1}, A_{65}^{2}$ and $A_{55}^{1}$ because

$$
\left\{\begin{array}{l}
A_{76}^{1}=6 A_{65}^{1} \\
A_{76}^{2}=6 A_{65}^{2}+5 A_{55}^{1} .
\end{array}\right.
$$

We get

$$
\left\{\begin{array} { l } 
{ A _ { 6 5 } ^ { 1 } = 5 A _ { 5 4 } ^ { 1 } = 5 \cdot 4 = 2 0 } \\
{ A _ { 6 5 } ^ { 2 } = 5 A _ { 5 4 } ^ { 2 } = 5 \cdot 7 + 4 \cdot 3 = 4 7 } \\
{ A _ { 5 5 } ^ { 1 } = 4 A _ { 4 4 } ^ { 1 } = 4 \cdot 3 = 1 2 }
\end{array} \Rightarrow \left\{\begin{array}{l}
A_{76}^{1}=6 \cdot 20=120 \\
A_{76}^{2}=6 \cdot 47+5 \cdot 12=342
\end{array}\right.\right.
$$

so, finally:

$$
C_{76}=A_{76}^{1} \cdot F_{3}+A_{76}^{2} \cdot F_{2}=120 \cdot 9+342 \cdot 2=1764 .
$$

For the last term, $C_{77}$, there is a single $A$ :

$$
C_{77}=A_{77}^{1} \cdot \underbrace{F_{2}}_{2}
$$

but

$$
A_{77}^{1}=6 \underbrace{A_{66}^{1}}_{5 A_{55}^{1}}=6_{(2)} ! \underbrace{A_{55}^{1}}_{4 A_{44}^{1}}=6_{(3)} ! \cdot 4=6_{(4)} !,
$$

which means that $C_{77}=6_{(4)} ! \cdot 2=6$ !, as we already knew.

\subsection{Matrix Calculation}

Of course, the calculus of a certain $C_{n k}$ becomes less fastidious if one previously (and automatically) calculates the several $A_{n k}^{i}$ by means of appropriate definitions and rules.

Let $\mathbf{A}_{k}$ be an infinite square matrix, where we'll put $A_{n k}^{i}$ for its entry in the supposed $n$-th row and the supposed $i$-th column; supposed because we'll make

$$
A_{n k}^{i}=A_{n_{0}+\Delta n ; k}^{i_{0}+\Delta i}
$$

where $\Delta n$ and $\Delta i$ are integers we'll call row and column increments respectively; $n_{0}$ and $i_{0}$ are the effective row and column numbers. For our purposes, we'll make $\Delta n=3$ and $\Delta i=0$; therefore, $A_{4 k}^{1}$ is the entry in the effective first row and first column.

We'll define $\mathbf{A}_{k+1}$ as the (first) diagonal descendant matrix of $\mathbf{A}_{k}$ - which is its diagonal ascendant - if

$$
A_{n ; k+1}^{i}=\sum_{j=1}^{i}(n-j) A_{n-j ; k}^{i-j+1} .
$$

This is an equivalent equation to (26). The idea is to present $\mathbf{A}_{5}$ as the descendant of $\mathbf{A}_{4}$ [the matrix corresponding to the table (25)]. Naturally, the second diagonal descendant of $\mathbf{A}_{k}$ is $\mathbf{A}_{k+2}$, the descendant of $\mathbf{A}_{k+1}$; generally speaking, $\mathbf{A}_{k+r}$ is the $\boldsymbol{r}$-th diagonal descendant of $\mathbf{A}_{k}$. 
So, if we define the matrix $\mathbf{A}_{4}$ following the equation (24), just like the table (25), in which we'll make all empty entries equal to zero, we get

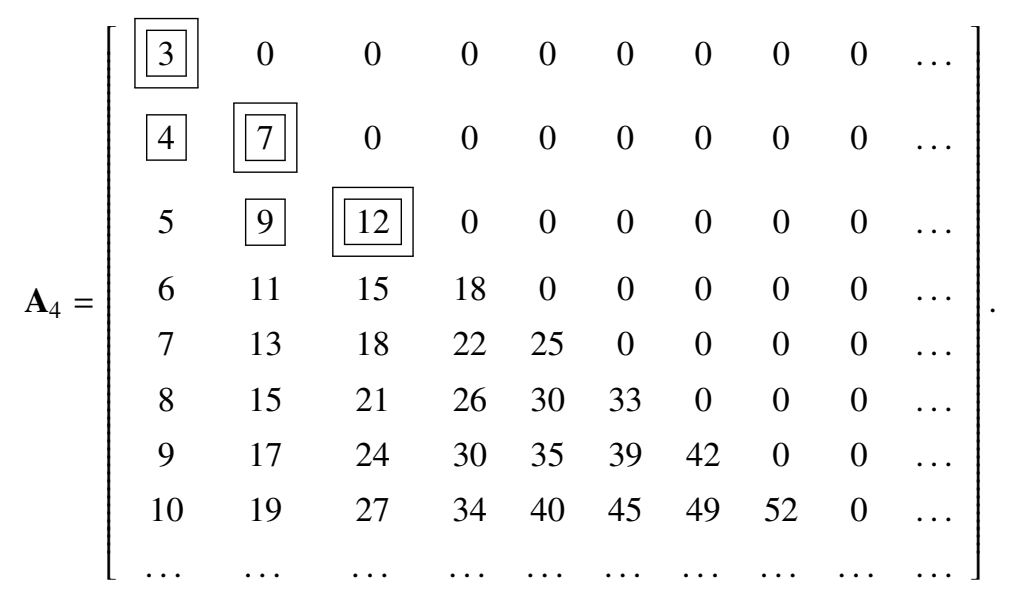

The first diagonal descendant of $\mathbf{A}_{4}$ is, then, the matrix

$$
\mathbf{A}_{5}=\left[\begin{array}{cccccccccc}
0 & 0 & 0 & 0 & 0 & 0 & 0 & 0 & 0 & \ldots \\
12 & 0 & 0 & 0 & 0 & 0 & 0 & 0 & 0 & \ldots \\
20 & 47 & 0 & 0 & 0 & 0 & 0 & 0 & 0 & \ldots \\
30 & 74 & 119 & 0 & 0 & 0 & 0 & 0 & 0 & \ldots \\
42 & 107 & 179 & 618 & 0 & 0 & 0 & 0 & 0 & \ldots \\
56 & 146 & 251 & 1361 & 29858 & 0 & 0 & 0 & 0 & \ldots \\
72 & 191 & 235 & 2688 & 6136 & 9616 & 0 & 0 & 0 & \ldots \\
90 & 242 & 431 & 4857 & 11702 & 19214 & 26036 & 0 & 0 & \ldots \\
\ldots & \ldots & \ldots & \ldots & \ldots & \ldots & \ldots & \ldots & \ldots & \ldots
\end{array}\right] .
$$

The modus operandi, for $A_{75}^{2}$ and $A_{75}^{3}$, as we saw before, is signalized respectively by the box and the double box on both matrices. Using the same simple routine, we obtain the second diagonal descendant:

$$
\mathbf{A}_{6}=\left[\begin{array}{cccccccccc}
0 & 0 & 0 & 0 & 0 & 0 & 0 & 0 & 0 & \ldots \\
0 & 0 & 0 & 0 & 0 & 0 & 0 & 0 & 0 & \ldots \\
60 & 0 & 0 & 0 & 0 & 0 & 0 & 0 & 0 & \ldots \\
120 & 342 & 0 & 0 & 0 & 0 & 0 & 0 & 0 & \ldots \\
210 & 638 & 1175 & 0 & 0 & 0 & 0 & 0 & 0 & \ldots \\
336 & 1066 & 2070 & 1361 & 22697 & 0 & 0 & 0 & 0 & \ldots \\
504 & 1650 & 3325 & 75841 & 265632 & 0 & 0 & 0 & 0 & \ldots \\
720 & 2414 & 5000 & 208899 & 815505 & 4240576 & 0 & 0 & 0 & \ldots \\
\ldots & \ldots & \ldots & \ldots & \ldots & \ldots & \ldots & \ldots & \ldots & \ldots
\end{array}\right]
$$

and so on. We see that the null rows increase from the top, one by one, as we 'descend' from the original matrix; related to that, at the same time the number of non-null entries diminishes in each row.

As an example, with a spreadsheet, we may use the matrix $\mathbf{A}_{6}$ to (automatically) compute $C_{76}$ and $C_{96}$ :

$$
\left\{\begin{array}{l}
C_{76}=\sum_{i=1}^{2} A_{76}^{i} \cdot F_{4-i}=120 \cdot 9+342 \cdot 2=1764 \\
C_{96}=\sum_{i=1}^{4} A_{96}^{i} \cdot F_{6-i}=336 \cdot 50+1066 \cdot 24+2070 \cdot 9+3135 \cdot 2=67284 .
\end{array}\right.
$$




\section{Stirling and Harmonic Numbers}

Some days after obtaining most of the precedent results (this is, except all the previous section), I learned about the so-called Stirling numbers. Then I found out that the arithmetic coefficients are equivalent to the unsigned Stirling numbers of the first kind $\left[\begin{array}{l}n \\ k\end{array}\right]$ as follows:

$$
\left[\begin{array}{l}
n \\
k
\end{array}\right]=C_{n ; n-k+1} \quad \text { or } \quad C_{n k}=\left[\begin{array}{c}
n \\
n-k+1
\end{array}\right]
$$

and that one may generate a triangle formed by these numbers, using a similar recurrence relation to the one established by theorem 1, which is essentially symmetrical to the Arithmetic Triangle!

I discovered these peculiar numbers - concerning permutations according to their number of cycles - by chance, related to the $n$-th harmonic number $H_{n}$, this is, the sum of the reciprocals of the first $n$ natural numbers:

$$
H_{n}=1+\frac{1}{2}+\frac{1}{3}+\cdots+\frac{1}{n}=\sum_{k=1}^{n} \frac{1}{k} .
$$

And I looked for harmonic numbers precisely because they corresponds to the sequence of the reciprocals of natural numbers and I had just proved the following theorem, which I found interesting.

Theorem 2. If $H_{n}$ is the $n$-th harmonic number, then, for $n>1$,

$$
H_{n}=\frac{C_{n+1 ; n}}{C_{n+1 ; n+1}}=\frac{C_{n+1 ; n}}{n !} .
$$

Proof. It is, once again, a proof by induction:

1) The equation is valid for $n=2: H_{2}=1+\frac{1}{2}=\frac{3}{2}=\frac{C_{32}}{C_{33}}$.

2) Suppose $H_{n-1}=\frac{C_{n ; n-1}}{C_{n ; n}}=\frac{C_{n ; n-1}}{(n-1) !}$.

3) Then,

$$
H_{n}=H_{n-1}+\frac{1}{n}=\frac{n C_{n ; n-1}+C_{n n}}{n !}=\frac{C_{n+1 ; n}}{C_{n+1 ; n+1}},
$$

according to theorem 1 .

For instance, $H_{5}=\frac{C_{65}}{C_{66}}=\frac{274}{120}=\frac{137}{60}=2,2833 \ldots$.

It has been proved that the harmonic series roughly approximate the natural logarithm function (Harmonic). In fact, it is quite simple to prove, using the MacLaurin-Cauchy integral test, that the series is divergent. But this means that

$$
\lim _{n \rightarrow \infty} H_{n}=\lim _{n \rightarrow \infty} \frac{C_{n ; n-1}}{C_{n n}}+\underbrace{\lim _{n \rightarrow \infty} \frac{1}{n}}_{0}=\infty \Rightarrow \lim _{n \rightarrow \infty} \frac{C_{n ; n-1}}{C_{n n}}=\infty,
$$

which is coherently obvious since the last quotient represents $H_{n-1}$. But one may also understand that this means that $C_{n ; n-1}$ grows faster then $C_{n n}$; and this is because of the double recurrence relation:

$$
\left\{\begin{array}{l}
C_{n ; n-1}=\underbrace{(n-1) C_{n-1 ; n-2}}_{\neq 0}+\underbrace{C_{n-1 ; n-1} ;}_{\neq 0} \text { and } \\
C_{n n}=\underbrace{(n-1) C_{n-1 ; n-1}}_{\neq 0}+\underbrace{C_{n-1 ; n}}_{0}=(n-1) C_{n-1 ; n-1}
\end{array} \Rightarrow \frac{C_{n ; n-1}}{C_{n ; n}}=\frac{C_{n-1 ; n-2}}{C_{n-1 ; n-1}}+\frac{1}{n-1} .\right.
$$

The last equation correspond to $H_{n-1}=H_{n-2}+\frac{1}{n-1}$, this is, (33) applied to $H_{n-1}$. But one clearly sees that there is a surplus of $\frac{1}{n-1}$ for $\frac{C_{n ; n-1}}{C_{n ; n}}$ in relation to $\frac{C_{n-1 ; n-2}}{C_{n-1 ; n-1}}$. This generically makes $C_{n ; n-1}$ grow faster then $C_{n n}$, with $n \rightarrow \infty$. On the other hand, the growth is attenuated as $n$ increases because the same happens with $1 / n$. 


\section{Further Matrix Representation}

If we form a lower triangular matrix of order $n$ with the $C_{i j}, 1 \leq i, j \leq n$,

$$
\mathbf{C}_{n}=\left[\begin{array}{ccccc}
1 & 0 & 0 & \ldots & 0 \\
1 & 1 & 0 & \ldots & 0 \\
1 & 3 & 2 & \ldots & 0 \\
1 & \ldots & \ldots & \ldots & \ldots \\
1 & C_{n 2} & C_{n 3} & \ldots & C_{n n}
\end{array}\right]
$$

then $\prod_{i=1}^{n} C_{i i}=(n-1) !^{*}$ is its determinant.

We may use a matrix representation for the first equation in (13), either in the 'individual' form (for each $n$ )

$$
\mathbf{P}_{n}=\mathbf{C}_{n n} \cdot \mathbf{A}_{n} \quad \text { this is, }\left[P_{n}\right]=\left[\begin{array}{llll}
C_{n 1} & C_{n 2} & \ldots & C_{n n}
\end{array}\right] \cdot\left[\begin{array}{c}
a^{n} \\
a^{n-1} d \\
\ldots \\
a d^{n-1}
\end{array}\right],
$$

or using the square matrix $\mathbf{C}_{n}$ above. For this purpose, however, we must introduce the concepts of progressive matrix and matrix column product.

1. A progressive matrix $\check{\mathbf{M}}_{n}$ on variables $x$ and $y$ is an upper triangular matrix of order $n$ defined by

$$
\left\{\begin{array}{l}
\check{M}_{i j}=x^{j-i} y^{i-1} \quad \text { for } j \geq i \\
\check{M}_{i j}=0 \quad \text { for } j<i .
\end{array}\right.
$$

Explicitly:

$$
\check{\mathbf{M}}_{n}=\left[\begin{array}{cccccc}
1 & x & x^{2} & x^{3} & \ldots & x^{n-1} \\
0 & y & x y & x^{2} y & \ldots & x^{n-2} y \\
0 & 0 & y^{2} & x y^{2} & \ldots & x^{n-3} y^{2} \\
0 & 0 & 0 & y^{3} & \ldots & x^{n-4} y^{3} \\
0 & \ldots & \ldots & \ldots & \ldots & \ldots \\
0 & 0 & 0 & 0 & \ldots & y^{n-1}
\end{array}\right]
$$

We see that the determinant of this matrix is given by $|\check{\mathbf{M}}|=\prod_{i=0}^{n-1} y^{i}=y^{\frac{n_{(2)} !}{2}}$.

Two special cases deserve attention:

(a) For $x=0$ and $y=1, \check{\mathbf{M}}_{n}$ turns into the identity matrix of order $n$.

(b) Making $x=y=1$, we obtain $\check{\mathbf{L}}_{n}$ :

$$
\begin{cases}\check{L}_{i j}=1 & \text { for } j \geq i \\ \check{L}_{i j}=0 & \text { for } j<i .\end{cases}
$$

2. Given two square matrices of the same order $n, \mathbf{B}$ and $\mathbf{A}$, the column product $\mathbf{B} \diamond \mathbf{A}$ is the $n \times 1$ matrix given by

$$
(\mathbf{B} \diamond \mathbf{A})_{i}=\sum_{j=1}^{n} B_{i j} A_{j i} .
$$

This means that each row $i$ of $\mathbf{B}$ doesn't multiply but the column of the same order $i$ of $\mathbf{A}$, thus producing a column matrix.

Now, coming back to the use of the Arithmetic Triangle in the form of $\mathbf{C}_{n}$, we'll make $x=a$ and $y=d$; furthermore, we'll multiply the matrix $\check{\mathbf{M}}_{n}$ by the initial term $a$, in such a way that we'll write for the $n \times 1$ matrix $\mathbf{P}_{n}$, which elements are $P_{i}(1 \leq i \leq n)$ :

$$
\mathbf{P}_{n}=\mathbf{C}_{n} \diamond a \cdot \check{\mathbf{M}}_{n},
$$


keeping in mind that

On the other hand:

$$
a \cdot \check{\mathbf{M}}_{i j}=\left[\begin{array}{c}
a^{j-i+1} \\
a^{j-i} d^{1} \\
a^{j-i-1} d^{2} \\
\cdots \\
a d^{j-1} \\
0 \\
\cdots \\
0
\end{array}\right] .
$$

$$
\mathbf{C}_{n} \diamond \check{\mathbf{L}}_{n}=[i !]_{n}
$$

As an example, let $a=2$ and $d=3$; well have the progression

$$
\begin{array}{lllllllllllll}
2 & 5 & 8 & 11 & 14 & 17 & 20 & 23 & 26 & 29 & 32 & 35 & \ldots
\end{array}
$$

and, for limit $n=6$,

$$
\mathbf{P}_{6}=\left[\begin{array}{cccccc}
1 & 0 & 0 & 0 & 0 & 0 \\
1 & 1 & 0 & 0 & 0 & 0 \\
1 & 3 & 2 & 0 & 0 & 0 \\
1 & 6 & 11 & 6 & 0 & 0 \\
1 & 10 & 35 & 50 & 24 & 0 \\
1 & 15 & 85 & 225 & 274 & 120
\end{array}\right] \diamond\left[\begin{array}{cccccc}
2 & 4 & 8 & 16 & 32 & 64 \\
0 & 6 & 12 & 24 & 48 & 96 \\
0 & 0 & 18 & 36 & 72 & 144 \\
0 & 0 & 0 & 54 & 108 & 216 \\
0 & 0 & 0 & 0 & 162 & 324 \\
0 & 0 & 0 & 0 & 0 & 486
\end{array}\right]=\left[\begin{array}{c}
2 \\
10 \\
80 \\
880 \\
12320 \\
209440
\end{array}\right] .
$$

Remark that these concepts of progressive matrix and matrix column product are quite useful, for example, to represent Newtons binomial, $S_{n}=(x+y)^{n}$, as

$$
\mathbf{S}_{n}=\mathbf{B}_{n+1} \diamond \check{\mathbf{M}}_{n+1},
$$

where $\mathbf{B}_{n+1}$ is the $(n+1) \times(n+1)$ matrix of the binomial coefficients $\left(\begin{array}{l}n \\ k\end{array}\right)=B_{n+1 ; k+1}$, called Pascal matrix of order $n+1$; or yet to express the equality, for $n>0$,

$$
Q_{n}=x^{n}-y^{n}=(x-y) \sum_{i=1}^{n} x^{n-i} y^{i-1}
$$

as

$$
\mathbf{Q}_{n}=[x-y]_{n} \diamond \check{\mathbf{M}}_{n}
$$

$[x-y]_{n}$ being the $n \times n$ matrix which elements are all equal to $x-y$.

\section{Conclusion}

The study of the product of the first $n$ terms of an arithmetic progression leads to an infinite numerical triangle - called Arithmetic Triangle - formed by certain coefficients, $C_{n k}$ and which may be progressively built. One discovers that the $C_{n k}$ correspond to the so-called Unsigned Stirling Numbers of the First Kind, which also generate a numerical triangle, essentially symmetrical to the one displaying the $C_{n k}$.

We established several proprieties and propositions concerning the Arithmetic Triangle and factorials. On the way, we generalized the concepts of factorial of order $k$, rising and falling factorials, and introduced the concepts of sum-factorial, descendant matrix, progressive matrix and matrix column product.

\section{Aknowledgment}

I wish to thank my pupil Tomás Pacheco for bringing up this issue and also for showing all the willingness and enthusiasm of youth. It is quite refreshing! 


\section{References}

Ferreira, Luís D. (2010). Integer Binomial Plan: a generalization on Factorials and Binomial Coefficients. Journal of Mathematics Research, 2(3), 18-35. https://doi.org/10.1016/0012-365X(84)90161-4

Broder, Andrei Z. (1984). The r-Stirling numbers. Discrete Mathematics 49(3), 241-259.

Deveci O., \& Akuzum Y. (2014), The Cyclic Groups and the Semigroups via MacWilliams and Chebyshev Matrices. Journal of Mathematics Research, 6(2), 55-58

Deveci O., \& Karaduman E. (2012), The cyclic groups via the Pascal matrices and the generalized Pascal matrices. Linear Algebra and its Applications, 437, 2538-2545.

Hiller, J. (2016). Old Friends in Unexpected Places: Pascal (and Other) Matrices in GLn(C). The American Mathematical Monthly, 161-167.

\section{Copyrights}

Copyright for this article is retained by the author(s), with first publication rights granted to the journal.

This is an open-access article distributed under the terms and conditions of the Creative Commons Attribution license (http://creativecommons.org/licenses/by/4.0/). 